LBL-35380

SLAC-PUB-6468

April 1994

$(\mathrm{T} / \mathrm{E})$

\title{
QCD and Intrinsic Heavy Quark Predictions for Leading Charm and Beauty Hadroproduction ${ }^{\star}$
}

\author{
R. Vogt \\ Lawrence Berkeley Laboratory \\ Berkeley, California 94720 \\ and \\ S. J. Brodsky \\ Stanford Linear Accelerator Center \\ Stanford University \\ Stanford, California 94309
}

\begin{abstract}
Recent experiments at Fermilab and CERN have observed a strong asymmetry between the hadroproduction cross sections of leading $D$ mesons, containing projectile valence quarks, and nonleading charmed mesons, without projectile valence quarks. The observed correlations of the $\pi^{ \pm} N \rightarrow D^{ \pm} X$ cross section with the projectile charge violates the usual assumption that heavy quark jet fragmentation factorizes. We examine the asymmetry between leading and nonleading charm production as a function of $x_{f}$ and $p_{T}^{2}$ assuming a two-component model combining leading-twist fusion subprocesses and charm production from intrinsic heavy quark Fock states. We predict a sizable asymmetry at low $p_{T}^{2}$ and high $x_{f}$ from coalescence of the charm quarks with the comoving spectator quarks of the projectile. An intrinsic $c \bar{c}$ production cross section of $0.5 \mu \mathrm{b}$ is sufficient to explain both the magnitude and kinematic dependence of the asymmetry. In contrast, the charm jet hadronization mechanisms contained in PYTHIA predict a sizeable leading charm asymmetry even at low $x_{F}$. The two-component model is extended to predict the asymmetry in $B$ meson production in proton-proton and pion-proton interactions.
\end{abstract}

\footnotetext{
${ }^{0} \star$ Supported in part by the U. S. Department of Energy under Contract Numbers DE-AC0376SF00515 and DE-AC03-76SF0098.
} 


\section{Introduction}

In leading-twist QCD, the factorization theorem [1] predicts that the fragmentation functions $D_{H / c}(z, Q)$ are independent of the quantum numbers of both the projectile and target. However, strong flavor correlations between the produced particle and the projectile have been reported in charm production [2, 3]. For example, in $\pi^{-}(\bar{u} d)$ interactions with hadrons or nuclei, the $D^{-}(\bar{c} d) x_{f}$ distribution is consistently harder than the $D^{+}(c \bar{d})$ distribution. The $D^{-}$and $D^{0}(c \bar{u})$ are referred to as "leading" charmed mesons while the $D^{+}$and $\bar{D}^{0}(\bar{c} u)$ are "nonleading". This leading behavior thus suggests that hadronization at large $x_{F}$ involves the coalescence of the produced charm or anticharm quarks with the spectator quarks of the projectile, just as in exclusive reactions. The study of this phenomena thus can provide new insights into the coherent mechanisms controlling the formation of hadrons in QCD.

Charmed hadron distributions are often parameterized as $\propto\left(1-x_{f}\right)^{n}$, where $n_{\text {nonleading }}$

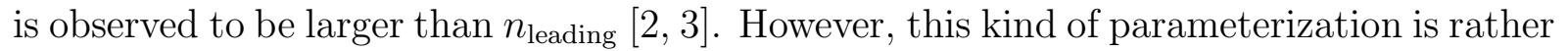
insensitive to the details of the mechanisms responsible for leading charm effects. A more sensitive observable, the asymmetry between leading and nonleading charm, has been used in the recent analyses of the WA82 [4] and E769 [5] collaborations. The asymmetry, defined as

$$
\mathcal{A}=\frac{\sigma(\text { leading })-\sigma(\text { nonleading })}{\sigma(\text { leading })+\sigma(\text { nonleading })},
$$

does not require the assumption of the functional form of the cross section. Both experiments find that the measured asymmetry $\mathcal{A}\left(x_{f}\right)$, integrated over $p_{T}$, increases from $\sim 0$ for $x_{f}$ near zero to $\sim 0.5$ around $x_{f}=0.65$ [4, 5]. However, the asymmetry $\mathcal{A}\left(p_{T}^{2}\right)$, integrated over all $x_{f}$, is found to be consistent with zero in the range $0<p_{T}^{2}<10 \mathrm{GeV}^{2}$ [5]. These facts are consistent if the leading charm asymmetry is localized at large $x_{F}$, involving only a small fraction of the total cross section.

The experiment WA82 measures charmed hadron production by $340 \mathrm{GeV} \pi^{-}$beams on $\mathrm{W} / \mathrm{Si}$ and $\mathrm{W} / \mathrm{Cu}$ targets [6]. Experiment E769 has measured $D^{ \pm}$production in $\mathrm{Be}, \mathrm{Al}$, $\mathrm{Cu}$, and $\mathrm{W}$ targets [7] with a mixed beam $\left(75 \% \pi^{-}\right.$and $\left.25 \% \pi^{+}\right)$at $250 \mathrm{GeV}$. The $D^{+}$is leading for a $\pi^{+}$projectile. In each experiment only $D^{ \pm}$mesons are used in the asymmetry analysis to avoid ambiguities in the assignment of leading and nonleading charm to $D^{0}$ mesons. The $D^{0}$, a leading charm state when directly produced by $\pi^{-}$beams, can also be produced by $D^{+\star}$ and $D^{0 \star}$ decays; e.g. $50 \%$ of nonleading $D^{+\star}$ 's decay to $D^{0}$.

Perturbative QCD at leading order predicts that $c$ and $\bar{c}$ quarks are produced with identical distributions. Next-to-leading order calculations do give rise to a small charge asymmetry $\left(\sim 10 \%\right.$ for $\left.x_{f} \sim 0.8\right)$ between $\bar{c}$ and $c$ production due to $q g$ and $q \bar{q}$ interference 8, 9]. However, this charge asymmetry should result in an increase of $D^{-}, \bar{D}^{0}$ production over $D^{+}$and $D^{0}$ at high $x_{f}$, not a separation between $D^{-}, D^{0}$ and $D^{+}, \bar{D}^{0}$.

How can one explain the origin of leading charm asymmetry within the context of QCD? It is clear that the produced charm (or anticharm) quark must combine with a projectile valence quark. Ordinary jet fragmentation (e.g. Peterson fragmentation [10]) cannot produce a leading particle asymmetry since it is independent of the initial state and thus the projectile valence quarks. This is an essential property of leading-twist factorization. However, one expects on physical grounds that a charm quark produced by 
fusion may coalesce with a comoving spectator valence quark [11, 12, 13, 14. For example, in QED, leptons of opposite charge moving with similar velocities can be captured into neutral atoms 15]. Since the capture is significant only at small relative rapidity, $\Delta y$, the effect on the total rate is higher twist.

In leading-twist QCD heavy quarks are produced by the fusion subprocesses $g g \rightarrow Q \bar{Q}$ and $q \bar{q} \rightarrow Q \bar{Q}$. The heavy $Q$ or $\bar{Q}$ normally fragments independently; however, there is a finite probability that it will combine with a spectator valence quark in the final state to produce a leading hadron. Coalescence is expected to dominate when the valence quark and the produced heavy quark have the same velocity. The coalescence amplitude should be largest at small relative rapidity since the invariant mass of the $\bar{Q} q$ system is minimal and the binding amplitude of the heavy meson wavefunction is maximal. This picture of coalescence is also consistent with "heavy quark symmetry" [16, 17]. A similar finalstate coalescence mechanism is contained in PYTHIA, a Monte Carlo program based on the Lund string fragmentation model [18]. Its string mechanism produces some charmed hadrons with a substantially larger longitudinal momentum than the charmed quarks originally produced by the fusion processes. At large $x_{f}$ and low invariant string masses, the produced $D^{-}$or $D^{0}$ inherits all the remaining projectile momentum while $D^{+}, \bar{D}^{0}$ production is forbidden. However, PYTHIA substantially overestimates the observed asymmetry $\mathcal{A}\left(x_{f}\right)$, particularly at low $x_{f}$. It also results in $\mathcal{A}\left(p_{T}^{2}\right) \sim 0.3$ for $0<p_{T}^{2}<10$ $\mathrm{GeV}^{2}$, overestimating the effect seen in the $x_{f}$-integrated data.

The difficulty that PYTHIA has in reproducing the shape of the leading charm asymmetry seems to be a characteristic feature of models based on final-state coalescence. If we parameterize the fusion-produced charm distribution as $d N_{c} / d x \propto(1-x)^{n}$, then the leading charmed hadron spectrum falls more slowly due to the extra momentum supplied by the projectile quark $d N_{D} / d x_{f} \propto\left[1-x_{f}(1-\delta)\right]^{n}$ where $\delta \sim m_{T_{\bar{q}}} / m_{T_{D}}$ and $m_{T}$ is the transverse mass. However, the asymmetries generated by this parameterization rise

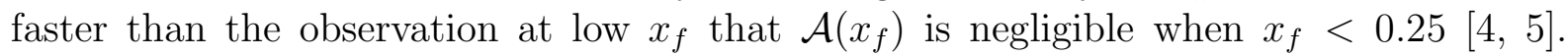
Thus a coalescence model strong enough to reproduce the hard spectrum of the leading charmed hadrons starting from the relatively soft fusion-produced charmed quarks tends to produce an excessivly large leading charm asymmetry over all phase space. It would be illuminating if the (true) rapidity distributions of the leading and nonleading charmed hadrons were measured to see whether or not their rapidity distributions track those of the underlying fusion-produced charmed quarks.

In the above picture of leading charm hadroproduction, it is implicitly assumed that coalescence is strictly a final-state phenomenon. In fact, the coalescence of the charm quark and a projectile valence quark may also occur in the initial state. For example, the $\pi^{-}$can fluctuate into a $|\bar{u} d c \bar{c}\rangle$ Fock state. The most important fluctuations occur at minimum invariant mass $\mathcal{M}$ where all the partons have approximately the same velocity. Characteristically, most of the momentum is carried by the heavy quark constituents of these Fock states. As viewed from the target rest frame, the intrinsic charm configurations can have very long lifetimes, of order $\tau=2 P_{l a b} / \mathcal{M}^{2}$ where $P_{l a b}$ is the projectile momentum. Intrinsic charm hadroproduction occurs dominantly when the spectator quarks interact strongly in the target [19], explaining why large $x_{f}$ charm production on nuclear targets is observed to have a strong nuclear dependence, similar to that of the inelastic hadronnucleus cross section. Since the charm and valence quarks have the same rapidity in an intrinsic charm Fock state, it is easy for them to coalesce into charmed hadrons and 
produce leading particle correlations at large $x_{f}$ where this mechanism can dominate the production rate. This is the basic underlying assumption of the intrinsic charm model 20].

The leading charm asymmetry must be a higher-twist effect or it would violate pQCD factorization. Final-state coalescence is higher twist since only a small fraction of the fusion-produced heavy quarks will combine with the valence quarks. Intrinsic heavy quark production is also higher twist-because the virtual configurations in the projectile wavefunction must be resolved during their limited lifetime. The cross section decreases with extra powers of $1 / m_{Q}$ relative to leading-twist fusion. From a general quantummechanical standpoint, both types of higher-twist mechanisms, coalescence of fusionproduced charm in the final state and coalesence of the intrinsic charm configurations in the initial state, must occur in QCD at some level.

In this paper, we shall calculate the asymmetry within a two-component model: parton fusion with coalescence, and intrinsic charm with valence-quark recombination [21.

\section{Leading-Twist Production}

The inclusive cross section for a single charmed hadron as a function of $x_{f}, x_{f}=$ $\left(2 m_{T} / \sqrt{s}\right) \sinh y$, and $p_{T}^{2}$ in leading twist QCD has the factorized form [21]

$$
\sigma=\frac{\sqrt{s}}{2} \int H_{a b}\left(x_{a}, x_{b}\right) \frac{1}{E_{1}} \frac{D_{H / c}\left(z_{3}\right)}{z_{3}} d z_{3} d y_{2} d p_{T}^{2} d x_{f},
$$

where $E_{1}$ is the energy of the charmed quark, $y_{2}$ is the rapidity of the charmed antiquark, $H_{a b}\left(x_{a}, x_{b}\right)$ is the convolution of the differential cross section with the parton distribution functions, and $m_{c}=1.5 \mathrm{GeV}$. We shall use the lowest-order parton fusion calculation. A $K$ factor of $\sim 2-3$ is included in the normalization of the fusion cross section. For consistency with the LO approximation, we use two current leading order sets of parton distribution functions, GRV LO [22] and Duke-Owens 1.1 [23] for the proton, and their pion counterparts, GRV LO [24] and Owens set 1 [25].

The fragmentation function, $D_{H / c}\left(z_{3}\right)$, describes the hadronization of the charmed quark where $z_{3}=x_{D} / x_{c}$ is the fraction of the charm momentum carried by the charmed hadron, assuming it is collinear with the charmed quark. We have studied two different fragmentation functions, a delta function, $\delta\left(z_{3}-1\right)$, and the Peterson function extracted from $e^{+} e^{-}$data [10]. We have shown previously that the Peterson function predicts a softer $x_{f}$ distribution than observed in hadroproduction, even at moderate $x_{f}$. The delta function model assumes that the charmed quark coalesces with a low- $x$ quark spectator from the sea (or a low momentum secondary quark produced in the collision) so that the charmed quark retains its momentum and velocity [21]. Either choice of fragmentation function is independent of the initial state and does not produce flavor correlations between the projectile valence quarks and the final-state hadrons.

In Fig. 1 we show the $x_{f}$ distributions calculated for (a) $\pi^{-} p$ and (b) $\pi^{+} p$ interactions at $250 \mathrm{GeV}$ and (c) $\pi^{-} p$ interactions at $340 \mathrm{GeV}$ using each set of structure functions with both choices of charm quark fragmentation function. The solid and dot-dashed curves give the calculated distributions using delta function and Peterson function fragmentation 
with GRV LO. The dashed and dotted curves illustrate the same calculations with DO 1.1. For charm production at these energies, DO 1.1 gives a somewhat larger cross section. As expected, delta function fragmentation results in harder distributions than those predicted by Peterson fragmentation for $x_{f}>0.2$. Note however that the conventional fusion model, even with delta function fragmentation cannot account for the shape of the leading $D$ production cross section from WA82 [⿴囗⿰丿㇄口]. (Since the normalization of the data has not been fixed, we normalize it to our calculated cross section.) The differences in the fusion cross sections from the GRV and DO distributions are only apparent at low $x_{f}$ where $g g \rightarrow c \bar{c}$ dominates since the gluon distributions are uncertain. At large $x_{f}$, where $q \bar{q} \rightarrow c \bar{c}$ is more important, little difference can be observed in the $x_{f}$ distributions because the valence distributions are relatively well measured. In the following we will use the GRV LO distributions only.

For valence quark coalescence to be effective as a leading charm production mechanism, either the $c$ or $\bar{c}$ must be comoving with the projectile valence quarks since capture into a bound state wavefunction favors constituents with similar rapidities. Is this possible in the fusion model? Most of the produced charmed quarks produced in the fusion reaction presumbably hadronize into charmed mesons or baryons independent of the projectile identity. To first approximation, the spectator quarks have the same rapidity as the projectile itself since they are bound state components of the projectile wavefunction. The produced heavy quarks tend to have low rapidity compared to the pion valence quarks. In the Lund string fragmentation model, as contained in PYTHIA [18, a charmed quark is always found at the endpoint of a string. This string pulls the charmed quark in the direction of the other string endpoint which is usually a beam remnant. When the two string endpoints are moving in the same general direction, the charmed hadron can then be produced at larger longitudinal momentum that then original charmed quark. In the extreme case where the string invariant mass is too small to allow the production of several particles, the string scenario reduces to a coalescence one, with the two string endpoints determining the flavor content of the produced hadron [26]. In Fig. 2(a) we compare the pion valence and sea quark rapidity distributions with the fusion-produced charmed quark rapidity distribution at $340 \mathrm{GeV}$. The solid and dotted curves are the pion valence and sea quark distributions while the dashed curve is the calculated charmed quark rapidity distribution. At this energy, the kinematic limit for charm production is reached at $y=2.8$. The spectator valence quarks tend to have larger rapidity than the charmed quarks. In the region where the distributions overlap, the sea quark rapidity density is also important. Thus we shall assume in this paper that charmed hadrons created from the leading twist fusion subprocesses arise dominantly either from independent fragmentation, coalescence with the projectile sea components, or coalescence with comoving secondary partons produced in the collision. Therefore we will also assume that the fusion mechanism produces a negligible asymmetry between $D^{+}$and $D^{-}$. We model the coalescence process with delta function fragmentation.

\section{Intrinsic Heavy Quark Production}

The fluctuation of a $\pi^{-}$into a $|\bar{u} d c \bar{c}\rangle$ Fock state produces a leading particle asymmetry through recombination of the intrinsic $c \bar{c}$ pair with the comoving valence quarks. The 
charmed quarks in the Fock state may be freed through soft interactions of the light valence quarks with the target [19]. The probability distribution corresponding to an $n$-particle Fock state (integrated over $k_{\perp i}$ ) is assumed to have the form

$$
\frac{d P_{\mathrm{ic}}}{d x_{1} \ldots d x_{n}}=N_{n} \alpha_{s}^{4}\left(m_{c \bar{c}}\right) \frac{\delta\left(1-\sum_{i=1}^{n} x_{i}\right)}{\left(m_{h}^{2}-\sum_{i=1}^{n}\left(\widehat{m}_{i}^{2} / x_{i}\right)\right)^{2}},
$$

where $\widehat{m}_{i}=\sqrt{\left\langle\vec{k}_{\perp i}^{2}\right\rangle+m_{i}^{2}}$ is the average transverse mass, $\left\langle k_{\perp}^{2}\right\rangle$ is proportional to the square of the quark mass, and $N_{n}$ is the normalization. Here we only consider the four-particle Fock state円. We have assumed the effective values $\widehat{m}_{q}=0.45 \mathrm{GeV}$ for the valence quarks and $\widehat{m}_{c}=1.8 \mathrm{GeV}$ for the charm quarks.

The $x$ distribution of intrinsic $c$ quarks in a pion is

$$
\frac{d P_{\mathrm{ic}}}{d x_{c}}=\int d x_{1} d x_{2} d x_{\bar{c}} \frac{d P_{\mathrm{ic}}}{d x_{1} \ldots d x_{c}}
$$

where $x_{1}$ and $x_{2}$ represent the valence quark momentum fractions. The intrinsic charm quarks hadronize both through fragmentation as in the parton fusion model and through coalesence with valence quarks. Fragmentation leads to distributions of the form

$$
\frac{d P_{\mathrm{ic}}^{F}}{d x_{D}}=\int d x_{c} d z_{3} D_{D / c}\left(z_{3}\right) \frac{d P_{\mathrm{ic}}}{d x_{c}} \delta\left(x_{D}-z_{3} x_{c}\right),
$$

where $x_{c}$ is the fraction of the projectile momentum carried by the $c$ quark and the definition of $z_{3}$ is identical to that in Eq. (2). The fragmentation of an intrinsic charm state itself does not produce a leading particle effect, since $D^{+}$and $D^{-}$production are equally likely.

The coalescence of one or both of the intrinsic charm quarks with comoving spectator valence quarks from an intrinsic charm state naturally produces leading charmed hadrons at large $x_{f}$. (By duality, one can think of the leading charmed mesons as preexisting in the intrinsic heavy quark Fock state.) The $\left|\pi^{-}\right\rangle=|\bar{u} d c \bar{c}\rangle$ state may coalesce into $|\bar{u} c\rangle$ and $|d \bar{c}\rangle, D^{0}$ and $D^{-}$, automatically producing leading charm since the charge conjugates contain no valence quarks. The leading $D^{-}$distribution is calculated from

$$
\frac{d P_{\mathrm{ic}}^{C}}{d x_{D^{-}}}=\int d x_{c} \frac{d P_{\mathrm{ic}}}{d x_{c}} \delta\left(x_{D^{-}}-x_{d}-x_{\bar{c}}\right)
$$

where the delta function defines $x_{D^{-}}$as the sum of the $\bar{c}$ and valence $d$ momentum fractions. In Fig. 2(b) we show the rapidity distributions for a light quark in a four particle intrinsic charm state (solid curve) and a charm quark (dashed curve). The $D^{-}$distribution from Eq. (6) is also shown (dot-dashed curve). The velocity (rapidity) of a heavy quark should remain unchanged by hadronization, up to order $\Lambda_{\mathrm{QCD}} / m_{Q}$. Thus there should be no acceleration of the heavy quark. However, since momentum is conserved, the hadron produced by the coalescence of equal rapidity partons will have the combined momentum of the heavy quark and valence spectator quark: $x_{D^{-}}=x_{q}+x_{\bar{c}}$. If coalescence dominantly

\footnotetext{
${ }^{1}$ Introducing additional light quarks or gluons reduces both the probability that the pion will fluctuate into this configuration and the probability that the $c \bar{c}$ will recombine with a valence quark to produce leading charm due to the presence of sea quarks.
} 
occurs when the combining partons have the lowest invariant mass, as in this model, then $x_{q} / x_{\bar{c}} \simeq m_{T_{q}} / m_{T_{\bar{c}}}$. In contrast, charmed hadrons produced by jet fragmentation have less momentum than the parent charmed quark. Thus it is natural that leading charmed hadrons created by the coalescence of a produced quark with a valence quark will have a harder longitudinal momentum distribution than nonleading charmed hadrons. This effect can perhaps also explain the pattern of leading strange-charmed baryons observed at large $x_{f}$ in the CERN WA62 hyperon beam experiment [27].

As in Ref. [21], we assume that the intrinsic charm model produces nonleading charm states by fragmentation of the intrinsic charm states and leading charm by both fragmentation and valence quark coalescence. Valence quark coalescence gives rise to the difference between the leading and nonleading distributions. The total charm distribution is then the sum of the parton fusion and intrinsic charm components, i.e. $d \sigma / d x_{f}=$ $d \sigma_{\mathrm{pf}} / d x_{f}+d \sigma_{\mathrm{ic}} / d x_{f}$.

Previously we obtained the normalization of the intrinsic-charm component by assuming that the ratio of the intrinsic charm cross section to the total charm cross section is identical to the ratio of the "diffractive" to the total $J / \psi$ production cross section measured by NA3, giving $\sigma_{\text {ic }} / \sigma_{c \bar{c}}^{\text {total }}=0.18$ for pion-induced production [28]. The constant $N_{4}$ was then fixed from this ratio under the assumption that the probability distribution and the differential cross section are identical [21]. However, because of the uncertainties in relating $\bar{c} c$ distributions to quarkonium production, it is difficult to use the $J / \psi$ cross section to obtain the absolute normalization of the intrinsic charm probability.

We set $N_{4}$ from the probability $P_{\text {ic }}$, with $P_{\text {ic }}=0.31 \%$ based upon calculations by Hoffmann and Moore [30] compared to EMC muoproduction data [29] and assume the same $P_{\text {ic }}$ for pion and proton projectiles. The intrinsic charm cross section will be proportional to the total inelastic cross section evaluated at $\hat{s}=\left(1-x_{f}\right) s$ since a soft interaction in the target breaks the coherence of the Fock state and brings the particles on shell [19, 31]. The cross section thus includes a resolving factor $\mu^{2} / 4 \widehat{m}_{c}^{2}$, so that

$$
\sigma_{\mathrm{ic}}=P_{\mathrm{ic}} \sigma_{\pi p}^{\text {in }} \frac{\mu^{2}}{4 \widehat{m}_{c}^{2}},
$$

where $\mu^{2}$ is a hadron scale parameter. Fixing $\mu^{2} \sim 0.2 \mathrm{GeV}^{2}$ from the NA3 ratio of 0.18 at $200 \mathrm{GeV}$ [28], we obtain $\sigma_{\text {ic }} \sim 1 \mu \mathrm{b}$ for a single charmed hadron with $x_{f}>0$ ]. (The intrinsic $c \bar{c}$ pair production cross section is $0.5 \mu \mathrm{b}$.) The nonleading and leading distributions are then

$$
\begin{aligned}
\frac{d \sigma_{\mathrm{ic}}^{N L}}{d x_{f}} & =\sigma_{\pi p}^{\mathrm{in}} \frac{\mu^{2}}{4 \widehat{m}_{c}^{2}} \frac{d P_{\mathrm{ic}}^{F}}{d x_{f}} \\
\frac{d \sigma_{\mathrm{ic}}^{L}}{d x_{f}} & =\sigma_{\pi p}^{\text {in }} \frac{\mu^{2}}{4 \widehat{m}_{c}^{2}}\left((1-\xi) \frac{d P_{\mathrm{ic}}^{F}}{d x_{f}}+\xi \frac{d P_{\mathrm{ic}}^{C}}{d x_{f}}\right),
\end{aligned}
$$

where $\xi$ is a parameter determining the relative importance of fragmentation and valence quark coalescence". Following Ref. [21], we have chosen $\xi=1 / 2$ in this analysis, except where otherwise noted.

\footnotetext{
${ }^{2}$ Our value of $\sigma_{\text {ic }}$ does not contradict the results of the E653 collaboration from $800 \mathrm{GeV} p \mathrm{Si}$ interactions [32], $\sigma_{\text {diff }}\left(D^{+}\right) / \sigma\left(D^{+}\right)<1.8 \%$. At $800 \mathrm{GeV}$, using $\sigma_{p p}^{\text {in }}$ in Eq. (7) and including an $A^{0.71}$ dependence for protons [28], we find $\sigma_{\text {ic }}(D) / \sigma(D) \sim 1.1 \%$.

${ }^{3}$ We have not made any distinction between the relative rates of charged and neutral $D$ production
} 
The nuclear dependence, $A$, of the fusion model is $A^{\alpha}$ where $\alpha \sim 1$ [33]. Since intrinsic charm production tends to occur on the nuclear surface [19], its target dependence is assumed to be $A^{\beta}$ where $\beta=0.77$ for pion-induced reactions [28]. The model thus predicts that leading charm effects are diminished in nuclear targets because of the relative suppression of the intrinsic charm component by $A^{\beta-1}$. This prediction is consistent with the $A$ dependence seen by the NA3 28] and E789 34 collaborations. We average our calculated asymmetry over the nuclear targets to compare with the data.

\section{Predictions of the Two-Component Model}

The nonleading and leading $x_{f}$ distributions from WA82 [4] (circles) and the combined $D^{ \pm}$data from E769 [7] (stars) are shown in Fig. 3(a) and (b) respectively. Because the data is given in arbitrary units, we give a common normalization to the calculations and data. Figure 3(a) shows the nonleading charm distributions for $\pi^{-} p$ interactions at 340 $\mathrm{GeV}$. The solid and dashed curves correspond to fusion calculations using delta function and Peterson function fragmentation respectively with the intrinsic charm contribution of Eq. (8). The Peterson function result lies well below the data, even at moderate $x_{f}$. Figure 3(b) gives our results for leading charm from $\pi^{-} p$ interactions at the same energy, including valence quark coalescence as well as fragmentation of the intrinsic charm quarks as in Eq. (9).

Figure 3(c) compares our results with the measured asymmetry at 250 (circles) and 340 $\mathrm{GeV}$ (stars) as well as the combined asymmetry from both experiments (squares) [4, 5]. The steepest increase of $\mathcal{A}\left(x_{f}\right)$ arises from Peterson function fragmentation (dashed curve) due to its much softer nonleading distribution at large $x_{f}$. The solid curve shows the delta function result. Because of the different nuclear target dependence for the intrinsic charm contribution, the leading charm effect diminishes in large nuclear targets. The dot-dashed curve shows delta function fragmentation without the average over nuclear target to show that the relative target dependencies of fusion and intrinsic charm do not strongly affect $\mathcal{A}\left(x_{f}\right)$. All the calculations based on the intrinsic charm model reproduce the general trend of the data. Increasing the coalescence contribution in Eq. (9) by increasing $\xi$ would increase the calculated asymmetry. The dotted curves in Fig. 3(b) and (c) show the effect of changing $\xi$ from $1 / 2$ to $9 / 10$ for a proton target. The leading $D x_{f}$ distribution is marginally changed, but the asymmetry is noticeably increased, bringing it into better agreement with the high $x_{f}$ data. This suggests that high statistics single $D$ distributions combined with the asymmetry data can fix relative normalization of the coalescence contribution. The leading $D^{-}$rapidity distribution from the two-component model is shown in Fig. 2(c) for both $\xi=1 / 2$ and $9 / 10$. Note that the total charm rapidity distribution is broader than the fusion result in Fig. 2(a), especially for $y>1$.

It should be emphasized that leading-twist QCD, including leading order corrections, does not produce an asymmetry between leading and nonleading charm [5, 9]. Both

but have rather assumed the same proportions for intrinsic charm and fusion. One could introduce an additional parameter in an attempt to model production differences i.e. between leading $D^{-}$and nonleading $\bar{D}^{0}$, both containing $\bar{c}$ quarks. Some differences in charged and neutral $D$ production naturally occur since $D^{\star}$ 's are commonly produced first but do not decay uniformly since $D^{\star+}$ and $D^{\star 0}$ both decay to $D^{0}$. See Ref. [13] for some discussion on relative $D^{-}, D^{0}$ production by fusion due to target effects. 
the intrinsic charm model and PYTHIA produce an asymmetry with $x_{f}$. In contrast to the PYTHIA predictions given in [4, 5], the intrinsic charm picture produces a slightly negative asymmetry for $x_{f}<0.4$. This is due to the difference between coalescence and fragmentation at low $x_{f}$. The $D$ meson $x_{f}$ distribution resulting from fragmentation, calculated using Eq. (5), peaks at $\left\langle x_{f}\right\rangle \sim 0.25$, while the coalescence of the valence quark with intrinsic charm quarks, Eq. (6), produces leading charm with $\left\langle x_{f}\right\rangle \sim 0.5$ [21]. When $x_{f}>0.4$, valence quark coalescence dominates over all other contributions, accounting for the rise in the calculated asymmetry. Without this intrinsic charm coalescence mechanism, i.e. $\xi=0$, there would be no asymmetry in this model. The asymmetry produced by intrinsic charm, shown in Fig. 3(c), corresponds to the single charm production cross section $\sigma_{\text {ic }} \sim 1 \mu \mathrm{b}$. At higher energies, as the fusion cross section increases, the relative intrinsic charm contribution to the charm production cross section will diminish since $\sigma_{\pi p}^{\text {in }}$ is nearly constant with energy. However, this will not affect the asymmetry at large $x_{f}$ until the fusion cross section dominates the intrinsic charm contribution.

The effects of intrinsic charm and coalescence occur dominantly at low $p_{T}$ where the valence and charm quarks are aligned. In order to illustrate this effect, we note that the $p_{T}$ dependence of these models is approximately

$$
\begin{aligned}
\frac{d N_{\mathrm{pf}}}{d p_{T}^{2}} & \propto \frac{\alpha_{s}^{2}\left(p_{T}^{2}+m_{c}^{2}\right)}{\left[p_{T}^{2}+m_{c}^{2}\right]^{2}} \\
\frac{d N_{\mathrm{ic}}}{d p_{T}^{2}} & \propto \frac{\alpha_{s}^{4}\left(p_{T}^{2}+m_{c}^{2}\right)}{\left[p_{T}^{2}+m_{c}^{2}\right]^{4}} .
\end{aligned}
$$

The higher-power falloff in the $p_{T}$ dependence of intrinsic charm reflects its higher-twist nature. The higher power of $\alpha_{s}$ appears in Eq. (11) because the gluon vertices with both the pion valence quarks and the intrinsic $c \bar{c}$ pair must be included in the calculation of the intrinsic charm amplitude, leading to $\alpha_{s}^{4}$ in the cross section. In the parton fusion model, the structure functions include the coupling of gluons and quarks to the incident hadrons, thus the fusion rate is proportional to $\alpha_{s}^{2}$.

The E769 collaboration has also investigated the $p_{T}^{2}$ dependence of the asymmetry [5]. Figure 4 shows our calculated $p_{T}^{2}$ dependence for several regions of $x_{f}$. Figure 4(c) compares the combined $D^{ \pm} p_{T}^{2}$ distribution from E769 in the range $0.1<x_{f}<0.7$ [7] with the model. The solid and dashed curves indicate delta function and Peterson function fragmentation respectively. We have assumed that fragmentation only affects the longitudinal momentum. The calculations are weighted by the percentage of the probability of intrinsic charm production from fragmentation and coalesence in each $x_{f}$ region. Nonleading and leading charm calculations are shown in Figs. 4(a) and (b). The solid and dashed curves show our delta and Peterson function results for $0<x_{f}<0.4$. Peterson function fragmentation, with its softer $x_{f}$ distribution, gives a somewhat larger $x_{f}$-integrated cross section when $0<x_{f}<0.4$ than the delta function. Conversely, when $0.4<x_{f}<1$, the harder distribution of delta function fragmentation (dot-dashed curves) gives it a larger $x_{f}$-integrated cross section than the Peterson function (dotted curves), both for nonleading and leading charm. The difference between the delta and Peterson function predictions at low $p_{T}^{2}$ in Fig. $4(\mathrm{~b})$ is due to the relative strength of valence quark coalescence in the forward region. As $p_{T}^{2}$ increases, the difference caused by the higher power falloff in $p_{T}^{2}$ is reduced primarily because of the higher power of $\alpha_{s}$ in Eq. (11). This difference will manifest itself most apparently in the asymmetry. 
In Fig. 5(a), we compare the $x_{f}$-integrated $\left(0.1<x_{f}<0.7\right)$ asymmetry of E769 [5] with our results. There is no asymmetry for $x_{f}>0$ because the integrated probabilities for leading and nonleading charm are equal. In the region covered by the data, there is a slight asymmetry, shown by the solid line for delta function fragmentation and the dashed line for the Peterson function. Both curves are multiplied by a factor of 50 to be visible. The choice of the delta function leads to a negative asymmetry due to its smaller integrated fragmentation component from Eq. (9). The Peterson function tends to make the intrinsic charm fragmentation component narrower, so that most of its contribution is contained in the region $0.1<x_{f}<0.7$, resulting in a positive $\mathcal{A}\left(p_{T}^{2}\right)$. For $0<x_{f}<0.4$, the calculated $\mathcal{A}\left(p_{T}^{2}\right)$ is negligibly small and negative and is not shown. When $0.4<x_{f}<1$, the resulting $\mathcal{A}\left(p_{T}^{2}\right)$ is larger and positive, as shown in Fig. 5(b). The solid curve shows delta function fragmentation, the dashed, Peterson function. Both decrease strongly with $p_{T}^{2}$, due in part to the different powers of the coupling constant in the distributions. Although the delta function is more physical in low $p_{T}^{2}$ hadroproduction, the Peterson function should take over at sufficiently large $p_{T}^{2}$ since it describes jet fragmentation in $e^{+} e^{-}$annihilation [35]. A nonzero $\mathcal{A}\left(p_{T}^{2}\right)$ is more likely to be found in the forward $x_{f}$ data at low $p_{T}^{2}$.

Recently, the Fermilab E791 collaboration has taken high precision charm production data with a $\pi^{-}$beam at $500 \mathrm{GeV}$ on carbon and platinum targets in the range $-0.1<x_{f}<0.8$ 36. We have calculated the $x_{f}$ and $p_{T}^{2}$ distributions and the corresponding asymmetries for a proton target for this experiment using GRV LO structure functions and delta function fragmentation. The fusion cross section increases by a factor of two between E769 and E791, decreasing the relative strength of the intrinsic charm contribution by $\sim 50 \%$ in the higher energy experiment. This reduces the asymmetry by $\sim 10 \%$ at $x_{f} \sim 0.8$ for E791. The $p_{t}^{2}$ dependence of the asymmetry is again the strongest for the forward $x_{f}$ region, $0.4<x_{f}<0.8$. (Below $x_{f} \sim 0.4 \mathcal{A}\left(x_{f}\right)$ is again slightly negative; the predicted asymmetry becomes positive for $x_{f}>0.4$.) We predict little change in $\mathcal{A}\left(p_{T}^{2}\right)$ between E769 and E791. The fusion cross section will continue to increase with incident energy while $\mathcal{A}\left(x_{f}\right)$ will correspondingly decrease, albeit more slowly than the intrinsic charm contribution to the total cross section.

\section{Predictions for Leading Beauty Hadroproduction}

We also use the two-component model to predict $B$ meson distributions. The parton distribution functions are more stable for $b \bar{b}$ production, thus our calculations with the GRV LO and DO 1.1 sets are very similar. We only show delta function fragmentation since there is less distinction between the Peterson and delta function predictions for $b$ quarks. One surprising prediction of our model is that a larger fraction of the inclusive $b$-quark cross section is produced from intrinsic beauty compared to the fraction of open charm produced by intrinsic charm at fixed energy. Of course, at fixed values of $\tau=$ $m_{Q \bar{Q}} / \sqrt{s}$, the fusion cross section decreases by $\sim\left(m_{c} / m_{b}\right)^{2}\left(\alpha_{s}\left(m_{b \bar{b}}\right) / \alpha_{s}\left(m_{c \bar{c}}\right)\right)^{2}$ between charm and beauty production. The normalization of the intrinsic heavy quark cross

section contains factors of $\widehat{m}_{Q}^{2} \alpha_{s}\left(m_{Q \bar{Q}}\right)^{4}$ so that the probability for producing intrinsic beauty relative to charm only decreases as $\left(\widehat{m}_{c} / \widehat{m}_{b}\right)^{2}$ rather than $\left(\widehat{m}_{c} / \widehat{m}_{b}\right)^{4}$ as may be 
expected from Eq. (3). An additional decrease of $\left(\widehat{m}_{c} / \widehat{m}_{b}\right)^{2}$ is associated with the resolving factor, $\mu^{2} / 4 \widehat{m}_{b}^{2}$. We assume $\widehat{m}_{b}=4.6 \mathrm{GeV}$ and take $\mu^{2}$ to be independent of both the projectile and the final state. Thus, the intrinsic beauty cross section is predicted to be

$$
\sigma_{\mathrm{ib}}=P_{\text {ic }}\left(\frac{\widehat{m}_{c}}{\widehat{m}_{b}}\right)^{2} \sigma_{\pi p}^{\text {in }} \frac{\mu^{2}}{4 \widehat{m}_{b}^{2}}\left(\frac{\alpha_{s}\left(m_{b \bar{b}}\right)}{\alpha_{s}\left(m_{c \bar{c}}\right)}\right)^{4}
$$

on the order of 2-3 nb, similar to the size of the fusion cross section at the energies we investigate. The nonleading and leading $B$ distributions are similar to those given in Eqs. (8) and (9). The fusion cross section is expected to increase rapidly with energy but $\sigma_{\mathrm{ib}}$ is proportional to the inelastic cross section. Therefore although $\sigma_{\mathrm{ib}} / \sigma_{b \bar{b}}^{\text {total }}$ is relatively large for our calculated distributions, it will decrease at higher energies.

No data on $B$ distributions or their associated asymmetries have yet been published. We have calculated $B$ production in $\pi^{-} p$ interactions at 250 and $340 \mathrm{GeV}$. Our predictions of the leading and nonleading distributions and the asymmetry between them is shown in Fig. 6. The solid lines in Figs. 6(a) and (c) illustrate the fusion mechanism with delta function fragmentation. The dashed curves show nonleading $B^{+}(\bar{b} u)$ production with intrinsic $b$ quark fragmentation as in Eq. (8) and the dot-dashed curves show leading $B^{-}(b \bar{u})$ 's as in Eq. (9). The difference between the fusion and the nonleading $B$ distribution is larger than for charm since $\sigma_{\mathrm{ic}}$ and $\sigma_{\mathrm{ib}}$ are coupled to the inelastic cross section. As the fusion cross section increases, $\sigma_{\mathrm{ib}} / \sigma_{b \bar{b}}^{\text {total }}$ is reduced, seen by comparing Figs. 6(a) and (c). In Figs. 6(b) and (d) we show the corresponding asymmetry calculations. The asymmetry is similar for charm and beauty because the shape of the intrinsic distribution is only weakly dependent on the heavy-quark mass.

Since fixed-target experiments can be done at Fermilab with an $800 \mathrm{GeV}$ proton beam, we include $p p$ predictions for $B$ production in Figs. 6(e) and (f) using the same notation as above. An intrinsic heavy quark state in the proton has at least a five quark configuration, thus a leading $B^{+}$or a $D^{-}$produced by valence quark coalescence has $\left\langle x_{f}\right\rangle \sim 0.4$ while intrinsic heavy quark fragmentation gives $\left\langle x_{f}\right\rangle \sim 0.2$, both smaller than in a pion projectile. The intrinsic heavy quark cross section with proton projectiles is somewhat larger since $\sigma_{p p}^{\text {in }}$ is $40 \%$ larger than $\sigma_{\pi p}^{\text {in }}$. The $x_{f}$ distribution from fusion is narrower in proton production, as shown in Fig. 6(e). A faster increase in the asymmetry is expected (see Fig. 6(f)) since $\left\langle x_{f}\right\rangle$ is reduced for the five-quark Fock state.

One interesting test of the extension of this model to $B$ production is the shape of the leading and nonleading distributions. Since $\sigma_{\mathrm{ib}}$ is comparable to the $b \bar{b}$ production cross section by fusion, a parameterization of the $x_{f}$ distribution as $\left(1-x_{f}\right)^{n}$ should give a harder distribution and smaller $n$ than expected from fusion production, especially for $p p$ production, measurable at current energies. As the incident energy increases, $n$ should increase as the fusion contribution becomes dominant. The difference between leading and nonleading $B$ distributions may become important for $\mathrm{CP}$-violation studies where an understanding of the symmetries of the $B$ hadroproduction cross section is crucial. This is particularly true for kaon beams in order to produce $B_{s}$ 's with a large Lorentz gamma factor. 


\section{Conclusions}

We have shown that the intrinsic charm model produces an asymmetry between nonleading and leading charmed hadrons as a function of $x_{f}$ and $p_{T}^{2}$. The asymmetry, $\mathcal{A}$, is predicted to be largest at low $p_{T}^{2}$ and to be an increasing function of $x_{f}$. The model also accounts for the shapes of the charmed hadron distributions in $x_{f}$. It would be interesting to look for an asymmetry between $\Lambda_{c}$ and $\bar{\Lambda}_{c}$ production both in $\pi^{-} p$ and $p p$ interactions. Since both $\Lambda_{c}$ and $\bar{\Lambda}_{c}$ contain a pion valence quark, the asymmetry should be small. However, the asymmetry could be large in $p p$ interactions where two of the proton valence quarks can coalesce with a charm quark to produce leading $\Lambda_{c}$ while the $\bar{\Lambda}_{c}$ should be centrally produced. We also note that charmed-strange mesons are produced at large $x_{f}$ by hyperon beams [27], a leading particle effect of the type studied here.

The asymmetry between $D^{-}$and $D^{+}$production in $p p$ collisions should also be checked. If the two-component model is correct, the asymmetry should begin to increase at lower $x_{f}$ than the calculated asymmetry with a pion projectile but should have approximately the same shape. We also predict that the $B$ and $D$ asymmetries should be similar. An additional check on our model comes from the shape of the $B$ distributions at large $x_{f}$, especially with a proton beam where the intrinsic beauty contributions should produce a broader distribution than expected from leading twist fusion subprocesses.

Acknowledgements We would like to thank J. A. Appel, J. Hewett, and P. Hoyer for discussions. We would also like to thank T. Sjöstrand for useful discussions about the Lund model and PYTHIA and for comments on the manuscript. 


\section{References}

[1] J. C. Collins, D. E. Soper and G. Sterman, Perturbative QCD, ed. A. H. Mueller (World Scientific, Singapore, 1989). G. Bodwin, Phys. Rev. D31 (1985) 2616, D34 (1986) 3932. J. Qiu and G. Sterman, Nucl. Phys. B353 (1991) 105,137.

[2] M. Aguilar-Benitez et al., Phys. Lett. 161B (1985) 400, Z. Phys. C31 (1986) 491.

[3] S. Barlag et al., Z. Phys. C49 (1991) 555.

[4] M. Adamovich et al., Phys. Lett. B305 (1993) 402.

[5] G. A. Alves et al., Phys. Rev. Lett. 72 (1994) 812.

[6] M. I. Adamovich et al., in proceedings of the 1989 International Symposium of Heavy Quark Physics, Ithaca, NY, 1989, Cornell Heavy Quark 1989:285.

[7] G. A. Alves et al., Phys. Rev. Lett. 69 (1992) 3147.

[8] W. Beenakker et al., Nucl. Phys. B351 (1991) 507.

[9] P. Nason, S. Dawson, and R. K. Ellis, Nucl. Phys. B327 (1989) 49.

[10] C. Peterson, D. Schlatter, I. Schmitt, and P. Zerwas, Phys. Rev. D27 (1983) 105.

[11] R. C. Hwa, Phys. Rev. D27 (1983) 653.

[12] S. J. Brodsky and J. F. Gunion, Phys. Rev. D17 (1978) 848.

[13] V. A. Bednyakov, Dubna preprint JINR E2-94-79 (1994), bulletin board hepph@xxx.lanl.gov-9403270.

[14] B. Andersson, G. Gustafson, and H.-U. Bengtsson, Lund preprint LU TP 83-4 (1983).

[15] S. J. Brodsky, J. F. Gunion, and D. E. Soper, Phys. Rev. D36 (1987) 2710.

[16] N. Isgur and M. B. Wise, in proceedings of Hadron '91, College Park, MD, 1991, College Park 1991, Proceedings, Hadron '91:549.

[17] W. E. Caswell and G. P. Lepage, Phys. Lett. 167B (1986) 437.

[18] H.-U. Bengtsson and T. Sjöstrand, Comput. Phys. Commun. 46 (1987) 43.

[19] S. J. Brodsky, P. Hoyer, A. H. Mueller, W.-K. Tang, Nucl. Phys. B369 (1992) 519.

[20] S. J. Brodsky, P. Hoyer, C. Peterson and N. Sakai, Phys. Lett. B93 (1980) 451; S.

J. Brodsky, C. Peterson and N. Sakai, Phys. Rev. D23 (1981) 2745.

[21] R. Vogt, S. J. Brodsky and P. Hoyer, Nucl. Phys. B383 (1992) 643.

[22] M. Glück, E. Reya, and A. Vogt, Z. Phys. C53 (1992) 127.

[23] J. F. Owens, Phys. Lett. B266 (1991) 126. 
[24] M. Glück, E. Reya, and A. Vogt, Z. Phys. C53 (1992) 651.

[25] J. F. Owens, Phys. Rev. D30 (1984) 943.

[26] T. Sjöstrand, private communication.

[27] S. F. Biagi et al., Z. Phys. C28 (1985) 175.

[28] J. Badier et al., Z. Phys. C20 (1983) 101.

[29] J. J. Aubert et al., Phys. Lett. 110B (1982) 73.

[30] E. Hoffmann and R. Moore, Z. Phys. C20 (1983) 71.

[31] S. J. Brodsky, J. C. Collins, S. D. Ellis, J. F. Gunion, and A. H. Mueller, in Proceedings of the Summer Study on the Design and Utilization of the Superconducting Super Collider, Snowmass, CO, 1984, edited by R. Donaldson and J. Morfin (Division of Particles and Fields of the American Physical Society, New York, 1985).

[32] K. Kodama et al., Phys. Lett. B316 (1993) 188.

[33] G. A. Alves et al., Phys. Rev. Lett. 70 (1993) 722.

[34] M. S. Kowitt et al., E789 Collaboration, FERMILAB-Pub-93/344, LBL-34790.

[35] P. Kesten et al., Phys. Lett. B161 (1985) 412.

[36] T. Carter, E791 collaboration, private communication. 


\section{Figure Captions}

Figure 1. The $x_{f}$ distributions for (a) $\pi^{-} p$ and (b) $\pi^{+} p$ interactions at $250 \mathrm{GeV}$ and (c) $\pi^{-} p$ interactions at $340 \mathrm{GeV}$ calculated from parton fusion. The solid and dot-dashed curves show calculations using GRV LO structure functions with delta function and Peterson function fragmentation. The dashed and dotted curves illustrate the same results using DO 1.1 structure functions. The leading $D^{-}$data from the WA82 collaboration [⿴囗十 are shown with the fusion calculation in (c).

Figure 2. The rapidity density of valence (solid curve) and sea quarks (dotted) in the GRV LO pion distributions [24 are compared with the rapidity density of the produced charm quarks (dashed) in (a). The valence (solid) and charm (dashed) rapidity distributions in an pion fluctuation into an intrinsic charm state are shown in (b), along with the resulting leading $D$ distribution from Eq. (6). In (c) we show our two-component model calculation including fusion with the leading charm distribution from Eq. (9). The model calculations with intrinsic charm have been converted to rapidity. All the calculations are for a $340 \mathrm{GeV} \pi^{-}$beam.

Figure 3. Our results for (a) nonleading charm and (b) leading charm distributions in $\pi^{-} p$ interactions at $340 \mathrm{GeV}$ and (c) the asymmetry compared with the WA82 [4] (circles) and E769 [5, 7] (stars) data. The combined asymmetry from both experiments is also shown (squares) [5]. The calculations are with GRV LO distributions using delta function (solid) and Peterson function (dashed) fragmentation with the intrinsic charm contributions to nonleading, Eq. (8), and leading, Eq. (9), charm production. The dotted curve in (b) shows the leading $D$ distribution with $\xi=9 / 10$ in Eq. (9). In (c), the dashed curve is calculated with the Peterson function and the solid curve with delta function fragmentation. Both are averaged over nuclear target. The dot dashed curve uses delta function fragmentation and a proton target. The dotted curve shows the leading contribution calculated with $\xi=9 / 10$ in Eq. (9) for a proton target.

Figure 4. Nonleading and leading charm $p_{T}^{2}$ distributions are shown in (a) and (b) for 250 $\mathrm{GeV} \pi^{-} p$ interactions. The solid and dashed curves are calculated using delta function and Peterson function fragmentation in the $0<x_{f}<0.4$ interval while the dot-dashed and dotted curves are calculated for $0.4<x_{f}<1$. The combined $D^{ \pm} p_{T}^{2}$ data from E769 [7] is compared to our calculation integrated over $0.1<x_{f}<0.7$ in (c). The solid and dashed curves use delta function and Peterson function fragmentation respectively.

Figure 5. The $x_{f}$-integrated $\left(0.1<x_{f}<0.7\right)$ asymmetry of E769 [5] is shown in (a). Our results, using delta (solid) and Peterson (dashed) fragmentation functions, are multiplied by a factor of 50 . The asymmetry in the forward region, $0.4<x_{f}<1$, is shown in (b). 
The solid and dashed curves are again our results for the delta and Peterson fragmentation functions.

Figure 6. Our predictions of leading and nonleading $B$ distributions are shown in (a), (c), and (e). The solid lines illustrate the fusion mechanism. The dashed curves show nonleading $B$ production with intrinsic $b$ quark fragmentation and the dot-dashed curves are leading $B^{\text {'s }}\left(B^{-}(b \bar{u})\right.$ for $\pi^{-}(\bar{u} d)$ and $B^{+}(u \bar{b})$ for $\left.p(u u d)\right)$. In (b), (d), and (f), we show the corresponding asymmetry calculations. 
This figure "fig1-1.png" is available in "png" format from: http://arxiv.org/ps/hep-ph/9405236v1 
This figure "fig2-1.png" is available in "png" format from: http://arxiv.org/ps/hep-ph/9405236v1 
This figure "fig1-2.png" is available in "png" format from: http://arxiv.org/ps/hep-ph/9405236v1 
This figure "fig2-2.png" is available in "png" format from: http://arxiv.org/ps/hep-ph/9405236v1 
This figure "fig1-3.png" is available in "png" format from: http://arxiv.org/ps/hep-ph/9405236v1 
This figure "fig2-3.png" is available in "png" format from: http://arxiv.org/ps/hep-ph/9405236v1 